ИЗВЕСТИЯ АКАДЕМИИ НАУК ЭСТОНСКОИ ССР. ФИЗИКА * МАТЕМАТИКА PROCEEDINGS OF THE ACADEMY OF SCIENCES OF THE ESTONIAN SSR. PHYSICS * MATHEMATICS

$1988,37,3$

\title{
ТЕМПЕРАТУРНАЯ ЗАВИСИМОСТЬ ЭКСИТОННЫХ СПЕКТРОВ ПОГЛОЩЕНИЯ. ВЫЧИСЛЕНИЕ НА ОСНОВЕ РЕКУРСИВНОГО МЕТОДА
}

\author{
(Представил В. Хижняков)
}

\section{1. Введение}

Теоретическому исследованию экситонных спектров поглощения диэлектрических кристаллов в различных предельных случаях посвящено большое число работ, выполненных с использованием различных приближений. Из них выделим: $\left[{ }^{1-3}\right]$, где предельные случаи слабой и сильной экситон-фононной связи исследовались на основе теории возмущений, $\left[{ }^{4,5}\right]$, в которых использовался метод моментов, $\left[{ }^{6-8}\right]$, авторы которых использовали различные варианты метода когерентного потенциала, $\left[{ }^{9}\right]$, где расчет проводился на основе адиабатического приближения, и $\left[{ }^{10}\right]$, в которой низкотемпературные спектры поглощения для различных наборов параметров исследовались на основе рекурсивного метода, использующего алгоритм Ланцоша $\left[{ }^{11,12}\right]$. Из работ, посвященных длинноволновым хвостам спектра поглощения и правилу Урбаха $\left[{ }^{13}\right]$ отметим $\left[{ }^{6,9,14}\right]$, в которых был предложен механизм формирования урбаховского хвоста, объясняющий ряд экспериментальных результатов, и $\left[{ }^{15}\right]$, интерпретирующую фононную структуру в низкотемпературных спектрах. Наиболее полное описание особенностей длинноволновых хвостов поглощения было получено в $\left[{ }^{8,16}\right]$.

Результаты названных работ ограничены определенной областью параметров либо, ввиду сделанных приближений, требуют дополнительной проверки иными методами. Поэтому представляет интерес исследовать спектр поглощения более общим методом, в значительной мере лишенным указанных недостатков и позволяющим в едином подходе описать наблюдающиеся здесь эффекты. Такая попытка предпринята в данной работе на основе модифицированного алгоритма Ланцоша $\left[{ }^{17}\right]$, являющегося, по существу, переформулировкой рекурсивного алгоритма Мори $\left[{ }^{18}\right]$, сводящей последний к виду, аналогичному обычному алгоритму Ланцоша $\left[{ }^{11}\right]$, и сохраняющей его положительные черты. Отметим, что идеи близкие к изложенным в $\left[{ }^{17}\right]$ высказывались в работах $\left[{ }^{19,20}\right]$. Алгоритм позволяет провести точное вычисление большого числа элементов цепной дроби, описывающей спектр поглощения, для произвольных параметров задачи, в том числе температуры кристалла. Исследование температурной зависимости спектра поглощения и является основной целью данной работы, 


\section{2. Описание модели и вычислительной процедуры}

Используемая в данной работе модель описывается гамильтонианом

$$
H=\varepsilon_{A} \sum_{n} a_{n}^{+} a_{n}-(B / 12) \sum_{n} \sum_{a}^{\prime} a_{n+a}^{+} a_{n}+\sum_{n} b_{n}^{+} b_{n}+\sqrt{S} \sum_{n} a_{n}^{+} a_{n}\left(b_{n}+b_{n}^{+}\right),
$$

где $a_{\mathrm{n}}^{+}$- оператор рождения френкелевского экситона на узле $\mathrm{n}$

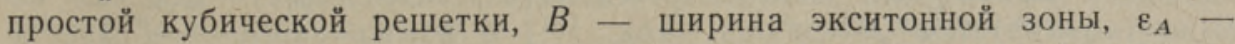
энергия центра зоны, отсчитываемая от основного состояния кристалла, штрих у суммы по а указывает, что суммирование проводится по 6 векторам ближайших соседей нулевого узла, $b_{\mathrm{n}}^{+}-$оператор рождения фонона на узле $\mathbf{n}$, частота фонона принята в качестве единицы частоты $(\hbar=1), S$ - стоксов сдвиг-выигрыш в энергии при колебательной релаксации возбуждения в случае $B=0$.

Спектр поглощения определяется формулой

$$
A(\Omega)=\pi^{-1} \operatorname{Re} R(-i \Omega+\eta),
$$

где

$$
R(z)=\int_{0}^{\infty} d t e^{-z t}\left\langle a_{x}(t) a_{x}^{+}\right\rangle
$$

$a_{\text {x }}=N^{-1 / 2} \sum_{\mathrm{n}} \exp (i x \mathrm{n}) a_{\mathrm{n}}-$ оператор уничтожения экситона с волновым вектором $\chi, N$ - число узлов в периодической области крнсталла, $a_{x}(t)$ - гейзенберговское представление оператора с гамильтонианом (1), $\Omega$ - частота поглощаемого фотона, $x$ - его волновой вектор, который далее будем полагать равным нулю, $\eta \rightarrow+0$, угловые скобки означают усреднение по каноническому ансамблю с гамильтонианом (1).

Для удобства ссылок приведем некоторые формулы из $\left[{ }^{17}\right]$. Если оператор $A_{0}$ удовлетворяет условию $\left\langle A_{0} A_{0}^{+}\right\rangle=1$, то процедура вычисления коррелятора $R(z)$ типа (3) с этим оператором, названная в $\left[{ }^{17}\right]$ модифицированным алгоритмом Ланцоша, сводится к построению набора операторов $A_{n}, n=1,2, \ldots$, удовлетворяющих условиям

$$
\left\langle A_{n} A_{n^{\prime}}^{+}\right\rangle=\delta_{n, n^{\prime}},
$$

в соответствии с рекуррентным соотношением

$$
\begin{aligned}
& {\left[H, A_{n}\right]=V_{n+1} A_{n+1}-E_{n} A_{n}+V_{n} A_{n-1},} \\
& E_{n}=-\left\langle\left[H, A_{n}\right] A_{n}^{+}\right\rangle, \quad V_{0}=0 .
\end{aligned}
$$

Получаемые в ходе этого построения величины $E_{n}, n=0,1, \ldots$, и $V_{n}$, $n=1,2, \ldots$, являются элементами цепной дроби, определяющей $R(z)$ :

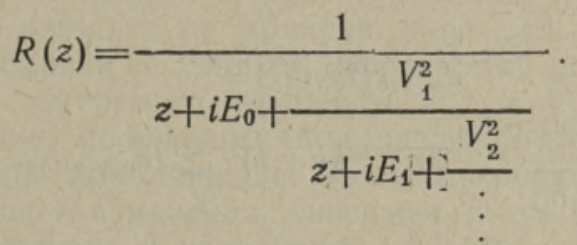

В обычной для диэлектрических кристаллов ситуации $\exp \left(-\varepsilon_{A} / T\right) \ll 1$, где $T-$ температура в энергетических единицах. Будем далее пренебрегать членами порядка или меньше этой экспоненты. В таком приближении $\left\langle a_{\varkappa} a_{*}^{+}\right\rangle=1$ и оператор $a_{\varkappa}$ можно непосредст- 
венно, без дополнительной перенормировки использовать в качестве $A_{0}$ в рекуррентной схеме (4), (5) (иная вычислительная процедура, не требующая выполнения условий нормировки $\left\langle A_{n} A_{n}^{+}\right\rangle=1$, описана в $\left[{ }^{17}\right]$. С точностью до указанных слагаемых спектр поглощения (2) нормирован на единицу. В рамках принятого приближения, как следует из (1) и (5), операторы $A_{n}$ можно представить в виде

$$
A_{n}=N^{-1 / 2} \sum_{v=0}^{n} \sum_{\mu=01}^{n-v} \sum_{v, \bar{m}_{\mu}, \mathbf{L}} D_{n, v, \mu}\left(\overline{\mathbf{I}}_{v} ; \overline{\mathbf{m}}_{\mu}\right) a_{\mathbf{L}} b_{\mathbf{L}+1}^{+} \ldots b_{\mathbf{L}++_{v}}^{+} b_{\mathbf{L}+\mathbf{m}_{1}} \ldots b_{\mathbf{L}+\mathbf{m}_{\mu}} \text {, }
$$

где введена сокращенная запись: $\overline{\mathbf{I}}_{v}$ обозначает $v$ аргументов $\mathbf{I}_{1}, \mathbf{I}_{2}, \ldots$ $\ldots, \mathrm{I}_{v}$, аналогично $\overline{\mathrm{m}}_{\mu}=\mathrm{m}_{1}, \mathrm{~m}_{2}, \ldots, \mathrm{m}_{\mu}$. Подставляя (7) в (4) и (5), находим

$$
\sum_{\gamma=0}^{n_{m}} \sum_{\delta=0}^{n_{m}-\gamma} \sum_{\gamma_{\gamma^{\prime}} \mathrm{m}_{\delta}} d_{n, \gamma, \delta}\left(\overline{\mathbf{l}}_{\gamma} ; \overline{\mathbf{m}}_{\delta}\right) d_{n^{\prime}, \gamma, \delta}\left(\overline{\mathbf{l}}_{\gamma} ; \overline{\mathbf{m}}_{\delta}\right)=\delta_{n, n^{\prime}}, \quad n_{m}=\min \left(n, n^{\prime}\right),
$$

$$
\begin{aligned}
& V_{n+1} d_{n+1, \gamma, \delta}\left(\overline{\mathbf{l}}_{\gamma} ; \overline{\mathbf{m}}_{\delta}\right)=\left(E_{n}-\varepsilon_{A}+\gamma-\delta\right) d_{n, \gamma, \delta}\left(\overline{\mathbf{l}}_{\gamma} ; \overline{\mathbf{m}}_{\delta}\right)+ \\
& +\frac{B}{12} \sum_{\mathbf{a}}^{\prime} d_{n, \gamma, \delta}\left(\overline{\mathbf{l}}_{\gamma}-\mathbf{a} ; \overline{\mathbf{m}}_{\delta}-\mathbf{a}\right)-\sqrt{\operatorname{Sn}(\gamma+1)} d_{n, \gamma+1, \delta}\left(\overline{\mathbf{l}}_{\gamma}, \mathbf{0} ; \overline{\mathbf{m}}_{\delta}\right)-
\end{aligned}
$$$$
-\sqrt{\frac{S(\bar{n}+1)}{\delta}} \sum_{i=1}^{\delta} \delta_{\mathrm{m}_{t}, 0} d_{n, \gamma, \delta-1}\left(\overline{\mathbf{I}}_{\gamma} ; \mathrm{m}_{\delta}^{i}\right)-
$$$$
-\sqrt{S(\vec{n}+1)(\delta+1)} d_{n, \gamma, \delta+1}\left(\overline{\mathbf{I}}_{\gamma} ; \overline{\mathbf{m}}_{\delta}, 0\right)-
$$

$-\sqrt{\frac{S \bar{n}}{\gamma}} \sum_{i=1}^{\gamma} \delta_{\mathbf{l}_{i}, 0} d_{n, \gamma-1, \delta}\left(\mathbf{1}_{\gamma}^{i} ; \overline{\mathbf{m}}_{\delta}\right)-V_{n} d_{n-1 \gamma, \delta}\left(\overline{\mathbf{l}}_{\gamma} ; \overline{\mathbf{m}}_{\delta}\right)$,

$$
E_{n}-\varepsilon_{A}=\sum_{\gamma=0}^{n} \sum_{\delta=0}^{n-\gamma} \sum_{\overline{1}_{\gamma}, \overline{\mathbf{m}}_{\delta}}(\delta-\gamma) d_{n, \gamma, \delta}^{2}\left(\overline{\mathbf{l}}_{\gamma} ; \overline{\mathbf{m}}_{\delta}\right)-
$$

$-\frac{B}{12} \sum_{\mathbf{a}}^{\prime} \sum_{\gamma=0}^{n} \sum_{\delta=0}^{n-\gamma} \sum_{\bar{\gamma}^{\prime}} \overline{\mathbf{m}}_{\delta} d_{n, \gamma, \delta}\left(\overline{\mathbf{l}}_{\gamma} ; \overline{\mathbf{m}}_{\delta}\right) d_{n, \nu, \delta}\left(\overline{\mathbf{l}}_{\gamma}-\mathbf{a} ; \overline{\mathbf{m}}_{\delta}-\mathbf{a}\right)+$

$+2 \sum_{\gamma=0}^{n-1} \sum_{\delta=0}^{n-\gamma} \sum_{\mathbf{i}_{\gamma}, \overline{\mathbf{m}}_{\delta}} \sqrt{S \bar{n}(\gamma+1)} d_{n, \gamma, \delta}\left(\overline{\mathbf{l}}_{\gamma} ; \overline{\mathbf{m}}_{\delta}\right) d_{n, \gamma+1, \delta}\left(\overline{\mathbf{l}}_{\gamma}, \mathbf{0} ; \overline{\mathbf{m}}_{\delta}\right)+$

$+2 \sum_{\gamma=0}^{n-1} \sum_{\delta=0}^{n-\gamma-1} \sum_{\mathbf{1}_{\gamma}, \mathbf{m}_{\delta}} \sqrt{S(\bar{n}+1)(\delta+1)} d_{n, \gamma, \delta}\left(\overline{\mathbf{1}}_{\gamma} ; \overline{\mathbf{m}}_{\delta}\right) d_{n, \gamma, \delta+1}\left(\overline{\mathbf{1}}_{\gamma} ; \overline{\mathbf{m}}_{\delta}, \mathbf{0}\right)$.

Здесь

$$
\left.=\sum_{\alpha=0}^{\alpha^{\prime}} \frac{(\delta+\alpha) !(\gamma+\alpha) !}{\alpha ! \sqrt{\gamma ! \delta !}} \frac{d_{n, \gamma, \delta}\left(\overline{\mathbf{l}}_{\gamma} ; \overline{\mathbf{m}}_{\delta}\right)=}{\sqrt{\bar{n}^{\gamma+2 \alpha}(\bar{n}+1)^{\delta}}} \sum_{\overline{1}_{\alpha}^{\prime}} D_{n, \gamma+\alpha, \delta+\alpha} \overline{(1}_{\alpha}^{\prime}, \overline{1}_{\gamma} ; \overline{\mathbf{l}}_{\alpha}^{\prime}, \overline{\mathbf{m}}_{\delta}\right),
$$

$\alpha^{\prime}$ - наибольшее целое, не превосходящее $(n-\gamma-\delta) / 2, \quad \bar{n}=$ $=[\exp (1 / \mathrm{T})-1]^{-1}, \overline{\mathbf{l}}_{\gamma}-\mathbf{a}=\mathrm{I}_{1}-\mathbf{a}, \mathbf{l}_{2}-\mathbf{a}, \ldots, \mathbf{l}_{\gamma}-\mathbf{a} ; \quad \mathbf{m}_{\delta}^{i}=\mathbf{m}_{1}, \ldots$ $\ldots, \mathbf{m}_{i-1}, \mathbf{m}_{i+1}, \ldots, \mathbf{m}_{\delta}$. Формулы (8)-(10) аналогичны формулам (10) - (12) из $\left[{ }^{17}\right]$, однако более удобны для вычислений. 
Величины $d_{n, \gamma, \delta}\left(\overline{\mathbf{I}}_{\gamma} ; \overline{\mathbf{m}}_{\delta}\right)$ инвариантны относительно перестановок любой пары векторов $\mathbf{l}_{i}$, а также любой пары векторов $\mathrm{m}_{i}$. Они инвариантны также относительно операций симметрии группы $O_{h}$, действующих одновременно на все векторы $\mathbf{I}_{i}$ и $\mathbf{m}_{i}$. Последнее является следствием симметрии задачи и начального оператора $A_{0}$, описываемого единственной ненулевой компонентой $D_{0,0,0}=d_{0,0,0}=1 \quad\left[{ }^{17}\right]$.

В $\left[{ }^{17}\right]$ отмечалась полная аналогия формул (4), (5) и следующих из них (8)-(10) соответствующим формулам алгоритма Ланцоша $\left[10,1^{12}\right]$, использовавшимся в $\left[{ }^{10}\right]$ для получения нольтемпературных спектров. Аналогична и последовательность вычислений: по найденным в предыдущем шаге компонентам $d_{n, \gamma, \delta}$ и $d_{n-1, \gamma, \delta}$ (в первом шаге - по известным компонентам $\left.d_{0, \gamma, \delta}\right)$ из $(9)$ и (10) получаем $E_{n}$ и $V_{n+1} d_{n+1, \gamma, \delta}$. Из условий ортогональности и нормировки (8) находим $V_{n+1}$, которую, как и в $\left[{ }^{10,12,17}\right]$, удобно выбрать действительной и положительной. Теперь можно определить $d_{n+1, \gamma, \delta}$ и повторить цикл вычислений для следующего значения $n$. Подчеркнем, что описанная процедура, исключая сделанное широко используемое при вычислении спектров поглощения приближение, во всех остальных отношениях точна. Впрочем, и от этого приближения можно отказаться, что приведет лишь к некоторому усложнению формул. Вычисления на каждом шаге единообразны и просты, причем требуют информации лишь о двух наборах компонент, $d_{n, \gamma, \delta}$ и $d_{n-1, \gamma, \delta}$. Объем резервируемой памяти $Ә \mathrm{BM}$ и количество операций можно существенно сократить, приняв во внимание указанные выше свойства симметрии компонент $d_{n, \gamma, \delta}-$ достаточно вычислять и запоминать лишь неодинаковые компоненты и кратность их повторения. Пронумеруем эти компоненты индексом $k$ (который, таким образом, объединяет индексы $\gamma, \delta$ и индекс, нумерующий неэквивалентные конфигурации $\left.\left(\overline{\mathbf{I}}_{\gamma} ; \overline{\mathbf{m}}_{\delta}\right)\right)$ и обозначим кратность повторения $N_{k}$.

Из (8)-(10) видно, что число ненулевых компонент с ростом $n$ растет и при заданном объеме памяти точные вычисления по описанному выше алгоритму при некотором $n$ придется прекратить. Чтобы иметь возможность продолжить счет далее, в нашей программе начиная с некоторого $n$ использовалась приближенная процедура, аналогичная предложенной в $\left[{ }^{10}\right]$ : после вычисления $V_{n}$ в соответствии с описанной выше точной процедурой, из полученных компонент $d_{n, k}$ отбирались $J$ компонент, обладающих наибольшей величиной произведения $d_{n, k}^{2} N_{k}$; затем они нормировались так, чтобы выполнялось условие нормировки $\sum_{k=1}^{J} d_{n, k}^{2} N_{k}=1 \quad($ см. 8)), и в дальнейшем считались единственными отличными от нуля компонентами. Таким образом, точный оператор базиса $\left\{A_{n}\right\}$ заменяется на приближенный, обладающий наибольшей проекцией на точный при отборе заданного количества компонент $J$. Разумеется, получаемые при таком расчете приближенные операторы $A_{n}$ теряют взаимную ортогональность. Однако, с одной стороны, как можно ожидать по крайней мере для некоторых областей параметров, они близки к точным операторам, а с другой - нас в большей степени интересуют величины $E_{n}$ и $V_{n}$. Сравнивая их и спектры поглощения, полученные при различных значениях $J$, можно проверить указанное приближение. Такая проверка показала, что при достаточно больших $J$ изменения, вносимые в эти величины при $n \leqslant 25$ и в спектры изменением $J$, становятся малыми и имеют такой же характер, как и в нольтемпературном случае (см. рис. 1,2 в $\left[{ }^{10}\right]$ ). Часть приводимых ниже спектров подвергалась такой проверке. При этом $J$ изменялось от 25 до 65.

Вычисления проводились до $n=25$. Қак и в нольтемпературном 
случае $\left[{ }^{10}\right]$, вычисленные зависимости $E_{n}$ и $V_{n}{ }^{2}$ при больших $n$ близки к линейным, на которые при некоторых значениях $S, B$ и $T$ накладываются осцилляции. Мы использовали это обстоятельство для экстраполяции величин $E_{n}$ и $V_{n}$ в область $n>25$ совершенно аналогично тому, как это делалось в $\left[{ }^{10}\right]$. Осцилляции с возрастающей с ростом $n$ амплитудой оказывают существенное влияние на спектр при $T \geqslant 1$ и $B=0$ (в случае $B=0$ вычислялось точно 40 пар величин $E_{n}$ и $V_{n}$ ). Найденные в этом случае, соответствующем модели ориентированного газа, спектры хорошо согласуются с известными результатами для примесного центра $\left[{ }^{21}\right]$. В этой связи отметим работу $\left[{ }^{22}\right]$, где для спектра поглощения примесного центра было найдено представление в виде ветвящейся цепной дроби. В случае же $B \geqslant S \geqslant 1, T \leqslant 1$, к которому относятся приводимые ниже спектры, сравнение спектров, полученных с линейной экстраполяцией и с экстраполяцией, где на линейный ход накладывались осцилляции с постоянной амплитудой, показало, что эти осцилляции слабо влияют на кривые. Использование более сложных экстраполяций, в частности с осцилляциями возрастающей амплитуды, представляется необоснованным как исходя из вида вычисленных зависимостей $E_{n}$ и $V_{n}^{2}$ в указанной области параметров, так и из-за относительно небольшого числа этих величин, полученных в расчете. Отметим однако, что наиболее существенные черты спектра (положение и относительная интенсивность наиболее интенсивных особенностей и в определенной мере их ширина) определяются в основном первыми звеньями цепной дроби $(6)$, элементы которых $E_{n}$ и $V_{n}$ вычисляются достаточно точно. Последующие члены дроби описывают более слабые особенности. Поэтому мы вправе ожидать, что полученные кривые верно отражают наиболее важные черты спектров поглощения (подробнее данный вопрос рассматривается в $\left.\left[{ }^{10}\right]\right)$.

В расчете приводимых ниже спектров использовалась линейная экстраполяция. Ее параметры определялись методом наименьших квадратов по 10-15 последним вычисленным значениям $E_{n}$ и $V_{n}^{2}$. Бесконечная цепная дробь (6) заменялась подходящей с числом звеньев до 20000 . Поскольку при такой замене спектр представляется набором $\delta$-функций $\left[{ }^{10,12}\right]$, для размытия несущественной структуры и представления спектра в виде непрерывной кривой использовалось их лоренцевское уширение: $\eta$ в (2) приписывалось конечное значение, которое в зависимости от вида спектра изменялось в пределах $0,01-0,3$.

\section{3. Форма спектра поглощения в центральной области}

Частоту поглощаемого фотона $\Omega$ будем отсчитывать от центра экситонной зоны. Спектр поглощения характеризуется 3 энергетическими параметрами: $B, S$ и $T$, измеряемыми в частотах фонона, принятых нами за единицу частоты. Как известно $\left[{ }^{7,10}\right]$, при $S \leqslant 1$ энергетический спектр экситон-фононной системы имеет черты, свойственные модели вибронного экситона при $B \lesssim 1$ и случаю слабой связи при $B \gg 1$. Изменение спектра с ростом температуры для параметров, попадающих в первую область, изображено на рис. 1 и для параметров из второй области - на рис. 2. Вид спектра и его температурное поведение в последнем случае сходны с получаемыми по теории возмущений $\left[{ }^{2}\right]$. Заметим, что при $T=0$ ширина главного максимума равна нулю для используемой модели экситон-фононного взаимодействия и для параметров рис. 2. Конечная ее величина на этом рисунке связана с принятой конечной величиной $\eta$. Уширение этого максимума с ростом $T$ по сравнению с $T=0$, как и красный сдвиг, обусловлено экситон-фононным взаимодействием. 


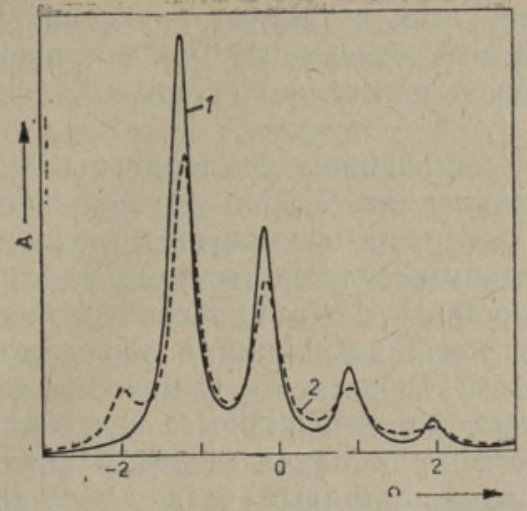

Рнс. 1. Спектр поглощения в случае $B=S=1$ при $T=0$ (кривая 1 ), и $T=0,5$ (2). Здесь и на следующих рисунках частота отсчитывается от центра экситонной зоны; расстояние между штрихами на оси абсцисс равно частоте фонона, принятой за единицу частоты.

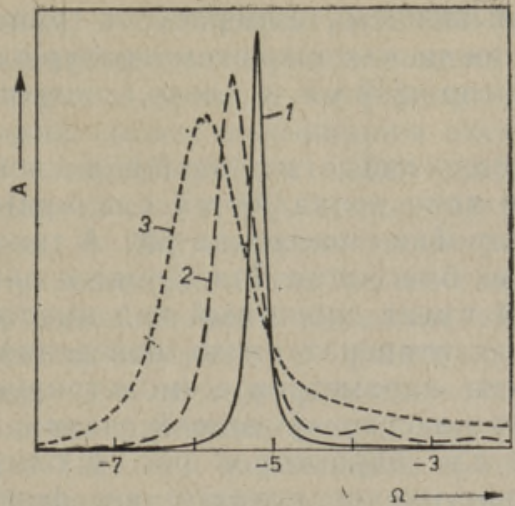

Рис. 2. Спектр поглощения в случае $B=10, S=1$ при $T=0$ (кривая 1 ), $T=1$ (2) и при $T=2$ (3). Чтобы получить более ясное представление о форме кривых их масштабы на оси ординат здесь и на рис. $3,4,6,6$ и 7 выбраны различными.

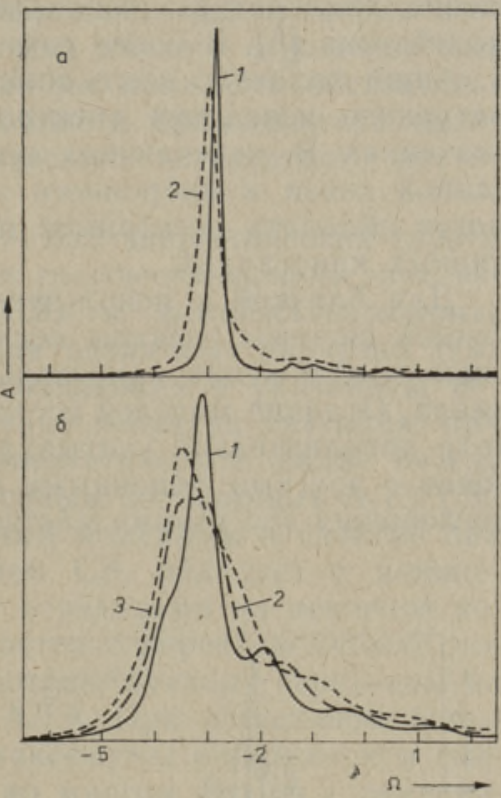

Рис. 3. Спектр поглощения в случае $B=5, S=1 ; a-T=0$ (кривая 1 ), $T=0,5 \quad(2) ; \sigma-T=1 \quad$ (кривая 1$)$, $T=1,25 \quad(2), T=1,37 \quad$ (3). исчезают различия между процессами поглощения с созданием лишь экситона и с созданием экситона при участии фонона. С дальнейшим ростом температуры низкочастотная часть этого максимума приобретает еще большую относительную интенсивность, а высокочастотная ослабляется, превращаясь при $T=1,37$ в плечо, которому можно сопоставить процесс с одновременным рождением экситона и фонона. Таким образом, в температурном интервале от $T=1$ до $T=1,37$ происходит изменение формы спектра, соответствующее изменениям в интерпретации его особенностей: плечо, при более низкой температуре отвечающее рождению экситона с поглощением фонона, 
с повышением температуры трансформируется в главный экситонный пик, тогда как низкотемпературный главный экситонный пик постепенно превращается в плечо, соответствующее рождению экситона и фонона.

Аналогичные изменения в спектре с изменением температуры еще более явно проявляются при меньших значениях $S$ (рис. 4). Характер перестройки спектра на рис. 4 такой же, как и на предыдущем рисунке, однако благодаря более узким пикам главный максимум при $T=1$ на рис. 4 имеет дублетный вид вместо столообразного. Еще одной особенностью температурного поведения спектров поглощения в указанной области параметров, демонстрируемой рис. 4, является изменение характера экситон-фононной связи с изменением температуры. Спектр при $T=0$ для параметров рис. 4 близок по виду к случаю слабой связи (на рисунке не показан; его форма близка к кривым для $T=0$ на рис. 2 и $3, a)$, тогда как кривые рис. 4 напоминают спектры вибронного экситона, и сравнительно узким и интенсивным максимумам в них можно, как и в случае вибронного экситона, поставить в соответствие связанные экситон-фононные состояния. Такое изменение характера спектра от одного предельного случая к другому с изменением температуры отчасти напоминает масштабный характер зависимости от параметров спектров в адиабатическом приближении. Здесь форма главного пика управляется единственным параметром - таким, что повышение температуры эквивалентно усилению экситон-фононного взаимодействия $\left[{ }^{9}\right]$. В более точном подходе, как видим, может изменяться и общий характер всего спектра. Описанные выше особенности температурного поведения спектров поглощения имеют место при $S \leqslant 1$ и значениях В, отвечающих промежуточной ситуации между случаями слабой связи и вибронного экситона. Такими наборами параметров могут обладать экситонные переходы в полупроводниковых и молекулярных кристаллах.

Для близкой к используемой в данной работе модели экситон-фононной системы спектры поглощения вычислялись в $\left[{ }^{8}\right]$ с использованием приближения одноузельного динамического когерентного потенциала. Отличие моделей состоит в том, что в $\left.{ }^{8}{ }^{8}\right]$ в стохастическом подходе дополнительно учитывалось взаимодействие ветви оптических фононов с другими фононными ветвями га счет ангармонизма. В спектре поглощения это взаимодействие приводит к уширению линий и в опре-

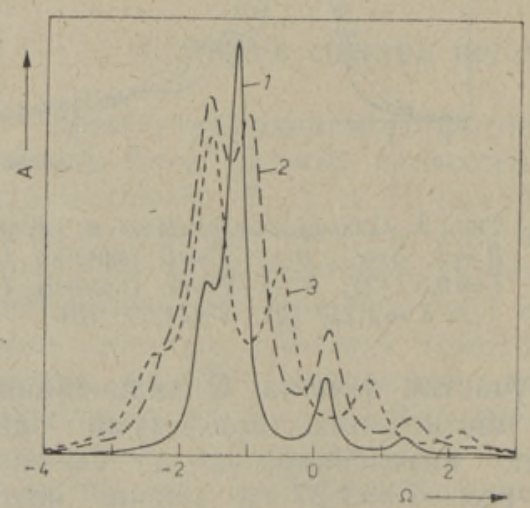

Рнс. 4. Спектр поглощения в случае $B=2, S=0,5$ при $T=0,5$ (кривая 1 ), $T=1 \quad$ (2) и $T=1,5$ (3).

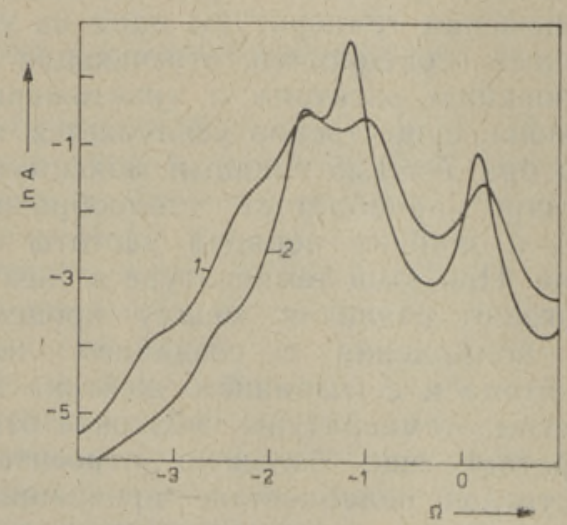

Рнс. 5. Спектр поглошения в случае $B=2, \quad S=0,5$ в полулогарифмическом масштабе при $T=1$ (I) и $T=0,5 \quad(2)$. 
деленном отношении сходно с уширением, вызываемым конечной величиной $\eta$ в (2). В частности, в $\left[^{8}\right]$ был найден спектр поглощения для тех же значений параметров, что и на рис. 4 (рис. $1, c$ в $\left[{ }^{8}\right]$; заметим, что параметр $B$ в этой работе, имеющий смысл полуширины экситонной зоны, вдвое меньше параметра $B$ в нашей работе). Для удобства сравнения две соответствующие кривые, как и в $\left[{ }^{8}\right]$, представлены в полулогарифмическом масштабе на рис. 5. Хотя кривые на этом рисунке и на соответствующем рисунке указанной работы во многом похожи (в особенности при $T=1$ ), обращает на себя внимание значительное различие в ширинах главных максимумов в спектрах при $T=0,5$ : максимум на рис. 5 не менее, чем на порядок шире своего аналога в $\left[{ }^{8}\right]$. Подчеркнем, что указанное различие не связано с выбором слишком большой величины $\eta$ в (2): при уменьшении $\eta$ от использовавшегося при получении рис. 5 значения ширина линии существенно не менялась. Вместо максимума на частоте - 1,6 в спектре при $T=0,5$ в соответствующем спектре указанной работы имеется плечо. Есть и другие, менее заметные различия. Причина указанных различий, по-видимому, связана с неадекватностью одноузельного приближения когерентного потенциала при описании главного экситонного пика и его окрестности в случае малых значений отношения $4 S(2 \bar{n}+1) / B^{2}$, отмеченной в $\left[{ }^{6}\right]$. В $\left[{ }^{6}\right]$, в частности, указывалось, что в этом случае данное приближение приводит к ширинам экситонных пиков, на порядок меньшим, чем полученные при частичном суммировании рядов теории возмущений в $\left[{ }^{2}\right]$. Отметим, однако, что величина данного отношения при $B=2, S=0,5, T=0,5$, равная 0,66 , не столь уж мала. Это обстоятельство указывает на еще большую узость области применимости одноузельного варианта приближения динамического когерентного потенциала для описания экситонного пика.

Как известно $[7,10,14,23]$, при $S \gg 1$ и $S \sim B / 2$ энергетический спектр экситон-фононной системы характеризуется сосуществованием двух типов возбуждений - почти свободных (СЭ) и автолокализованных (АЛЭ) экситонов. Спектры поглощения для различных значений температуры и материальных параметров, попадающих. в указанную область, приведены на рис. 6. На этом рисунке показана ситуация, при которой Г-точка зоны СЭ находится на энергетической шкале между основным и первым-колебательно-возбужденным состояниями АЛЭ (см. также $\left.\left[{ }^{10}\right]\right)$. В соответствии с этим, главный максимум в спектре при $T=0$, отвечающий переходам с рождением СЭ, обладает с длинноволновой стороны плечом, обусловленным переходами на основное колебательное состояние АЛЭ, и сопровождается с коротковолновой стороны серией более слабых максимумов, соответствующих переходам на более высокие колебательные состояния АЛЭ. При небольшом повышении температуры $(T=0,1)$ последние максимумы уширяются и смещаются в красную сторону. Этот эффект во многом сходен с температурными уширениями и сдвигами линий в спектрах примесных центров с квадратичным вибронным взаимодействием [21]. Действительно, как видно из спектра при $T=0$, расстояние между указанными максимумами несколько меньше частоты фонона - результат, к которому в случае примесного центра приводит квадратичное вибронное взаимодействие, а в рассматриваемой задаче с линейным экситон-фононным взаимодействием - конфигурационное смешивание состояний СЭ и АЛЭ $\left[{ }^{7,10,14}\right]$. В результате, наложение линий, связанных при конечной температуре с переходами из различных колебательных состояний основного электронного состояния, и приводит к указанным сдвигам и уширениям. Обратим внимание, что максимум СЭ и его длинноволновое плечо остаются практически неизменными в температурном интервале от 0 до 0,1 . Таким образом, температурное поведение особеннос- 
тей спектра, связанных с СЭ и АЛЭ, различно, что отражает различие в соответствующих механизмах - в случае СЭ температурные изменения связаны не с изменением относительной роли переходов между практически неизменными элементами энергетической диаграммы, как в случае АЛЭ или примесного центра, а с изменением свойств соответствующего элементарного возбуждения. Полученное направление температурного сдвига максимумов АЛЭ обусловлено избранной моделью с линейным экситон-фононным взаимодействием. Хорошо, однако, известно, что в релаксированном состоянии деформация решетки в окрестности АЛЭ велика и для адекватного его описания могут понадобиться более высокие члены разложения (см., напр., $\left.\left[{ }^{24,25}\right]\right)$. Это приводит к существенному изменению колебательного спектра в окрестности АЛЭ, и в результате сдвиги могут оказаться не красными, а синими. Температурное поведение максимумов СЭ и АЛЭ и в этом случае должно быть различным.

При дальнейшем повышении температуры указанная фононная структура быстро размывается (рис. 6,6$)$ и спектры принимают вид близкий к полученному в $\left[{ }^{1,2}\right]$ с тем, однако, существенным отличием, что на коротковолновом спаде спектров при $T=0,5$ и 1 имеется хорошо заметное плечо. Это плечо, начинаясь приблизительно на частоту фонона выше центра максимума СЭ (см. также рис. 7,6$)$, по своим спектральным проявлениям аналогично соответствующим особенностям в спектрах при $S \leqslant 1$, когда состояний АЛЭ нет (напр., спектр при $T=1,37$ на рис. 3). Поэтому его естественно связать с переходами с рождением СЭ и одного или нескольких фононов. В этом также проявляются различия в температурном поведении максимумов СЭ и АЛЭ.

Параметрам рис. 7 отвечает ситуация, когда автолокализованные состояния не возникают. Упоминавшееся выше коротковолновое плечо в высокотемпературных спектрах здесь более заметно.

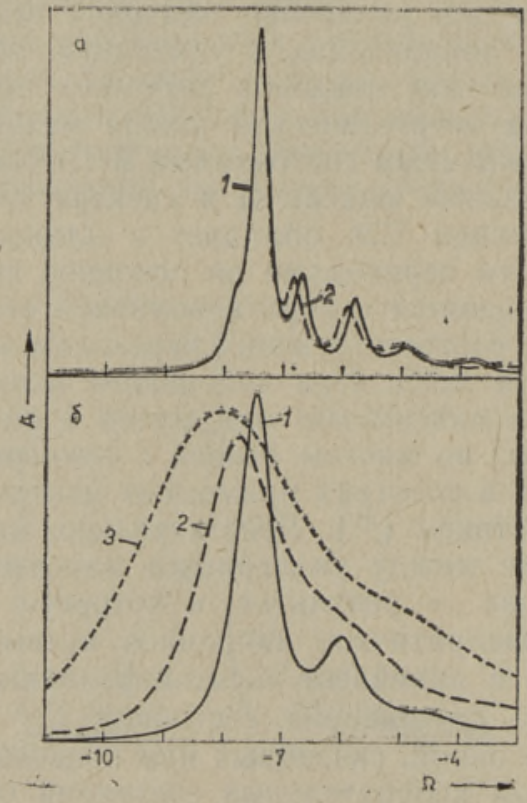

Рис. 6. Спектр поглощения в случае $B=12, S=7 ; a-T=0$ (кривая 1 ), $T=0,1 \quad(2) ; \sigma-T=0,25 \quad(1), T=$ $=0,5 \quad(2), T=1 \quad(3)$.

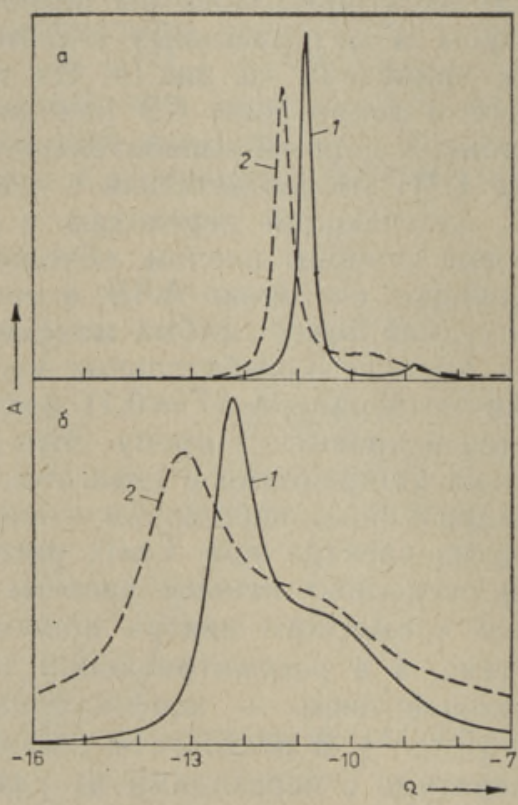

Рнс. 7. Спектр поглощения в случае $B=20, S=7 ; a-T=0$ (кривая 1 ), $T=0,5$ (2): $\check{\sigma}-T=1$ (1), $T=2$ (2). 
Таким образом, фононная структура, связанная с АЛЭ, может наблюдаться в спектре поглощения лишь при достаточно низких температурах. Имеется большое число экспериментальных работ, выполненных в основном на щелочно-галоидных кристаллах прн температурах, близких к температуре жидкого гелия, в которых в окрестности главного пика была обнаружена структура, напоминающая полученную в данной работе и в $\left[{ }^{10}\right]$. По-видимому, первыми экспериментальными результатами такого рода являлись спектры отражения и $\varepsilon_{2}$, приведенные в $\left[{ }^{26,27}\right]$. Дублетный характер нижайшего экситонного пика в спектрах отражения кристаллов КІ при $6 \mathrm{~K}$ из $\left[{ }^{26}\right]$ и $\mathrm{CsBr}$ при $4,2 \mathrm{~K}$ из $\left[{ }^{28}\right]$ сходен со спектром в случае $B=14, S=7$ из $\left[{ }^{10}\right]$, а приблизительно эквидистантная структура с шагом близким к частоте оптического фонона в спектре LiI при $6 \mathrm{~K}$ из $\left[{ }^{26}\right]$ сходна с нольтемпературным спектром из данной работы (рис. 6). Спектр $\varepsilon_{2}$ кристалла $\mathrm{LiBr}$ при $55 \mathrm{~K}\left[{ }^{27}\right]$ похож на спектр при $T=0,25$ на рис. 6 . Отклонения от формы главного пика типа асимметричного лоренциана работы $\left[{ }^{2}\right]$ на коротковолновом спаде спектра были зарегистрированы при температурах, близких к температуре жидкого азота в $\mathrm{CsBr}\left[{ }^{28}\right], \mathrm{KI}, \mathrm{KBr}$ и $\mathrm{KCl}\left[{ }^{29}\right]$. В последней работе было показано, что эти отклонения имеют вид плеча, начинающегося на частоту LO-фонона выше частоты максимума главного пика. Такая форма спектра похожа на кривые на рис. 6,6 и 7,6 , хотя указанное плечо на этих рисунках выражено заметно сильнее, чем в эксперименте. Это, по-видимому, связано с тем, что взаимодействие с акустическими фононами и другими оптическими ветвями колебаний приводит к некоторому сглаживанию указанной особенности спектра.

Представляет интерес сравнить полученные в данной работе шири ны главных максимумов (на половине их высоты) и их температурные сдвиги с аналогичными параметрами из $\left[{ }^{9}\right]$, полученными для трехмерного кристалла в адиабатическом приближении. Поскольку результаты этих работ относятся к случаю достаточно высоких температур, для сравнения использовались кривые, отвечающие этому условию $(B=10$, $S=1, T=1$ и $2 ; B=5, S=1, T=1,37 ; B=20, S=7, T=2$ и $1 ; B=12$, $S=7, T=0,5$ и 1). При проведении сравнения учитывалась отмеченная в $\left[{ }^{9}\right]$ возможность скалирования спектральных кривых, полученных в адиабатическом приближении. Ширины главных максимумов для указанных наборов параметров оказались близкими к найденным в $\left[{ }^{9}\right]$. В адиабатическом приближении положение главного максимума с понижением температуры стремится к значению $-B / 2$. Температурные сдвиги, отсчитываемые от этой частоты, также близки к полученным в [ $\left.{ }^{9}\right]$. Однако естественнее отсчитывать эти сдвиги от реального положения максимума при $T=0$, отличающегося от $-B / 2$ на величину поляронного сдвига. Различие в значениях сдвигов, полученных двумя указанными способами, может быть весьма существенным (см., напр., рис. 6 , спектры при $T=0,5$ и 1).

\section{4. Длинноволновой хвост оптического поглощения}

В большом числе диэлектрических, молекулярных и полупроводниковых кристаллов частотное поведение длинноволновых хвостов оптического поглощения при достаточно высоких температурах удовлетворяет эмпирическому правилу (правилу Урбаха)

$$
A(\Omega)=A \exp \left\{-\sigma \frac{2\left(\Omega_{\theta}-\Omega\right)}{\omega} \operatorname{th}\left(\frac{\omega}{2 T}\right)\right\},
$$

где $\omega$ - эффективная фононная частота, $\Omega_{0}$ - частота конвергенции, как правило, близкая к частоте главного максимума спектра при $T=0$, 
б - коэффициент наклона. Два последних параметра обычно слабо зависят от температуры. При более низкой температуре в некоторых полупроводниковых и молекулярных кристаллах на длинноволновом спаде спектра выделяется фононная структура с шагом примерно равным частоте фонона. В кристаллах с более сильной экситон-фононной связью, например, щелочно-галоидных, такая структура не наблюдается и при низких температурах. Другим отличием этих кристаллов от молекулярных и полупроводниковых является конечное поглощение в области ниже максимума СЭ при температурах близких к нулю. Подробное обсуждение экспериментальных работ по правилу Урбаха в оптических спектрах можно найти в $\left[{ }^{13}\right]$.

На рис. 5 в полулогарифмическом масштабе приведен спектр поглощения для материальных параметров, соответствующих слабой экситон-фононной связи, реализующейся, в частности, в полупроводниковых кристаллах. На длинноволновом спаде спектра при $T=1$ хорошо видна фононная структура с шагом примерно равным частоте фонона, которая для данного набора параметров отчасти сохраняется и при $T=1,5$ (одна из таких особенностей в этом спектре видна на рис. 4). Указанная структура, как уже отмечалось, связана с оптическими переходами с рождением экситона и уничтожением одного или нескольких фононов и может быть интерпретирована в рамках теории возмущений $\left[{ }^{15}\right]$. На рис. 3, б видно, как при ином наборе параметров происходит размытие одной из особенностей в аналогичной фононной структуре с повышением температуры. При понижении температуры до $T=0$ оптическое поглощение на частотах ниже экситонного максимума исчезает (см., напр., рис. 3 ; конечное поглощение на частотах ниже максимума в спектре при $T=0$ здесь связано с конечной величиной $\eta$ в (2) и стремится к нулю при $\eta \rightarrow 0$ ).

Иная ситуация в случае $S \gg 1$ и $S>B / 2$, реализующемся, в гастности, в щелочно-галоидных кристаллах. В этом случае, как видно из рис. 6 , и при $T=0$ имеется конечное поглощение на частотах ниже максимума СЭ, связанное с переходами на колебательные состояния АЛЭ (в данном случае на нулевой колебательный уровень; длинноволновое плечо в спектре при $T=0$ на рис. 6). Этот механизм конечного длинноволнового поглощения в низкотемпературных спектрах идеаль-

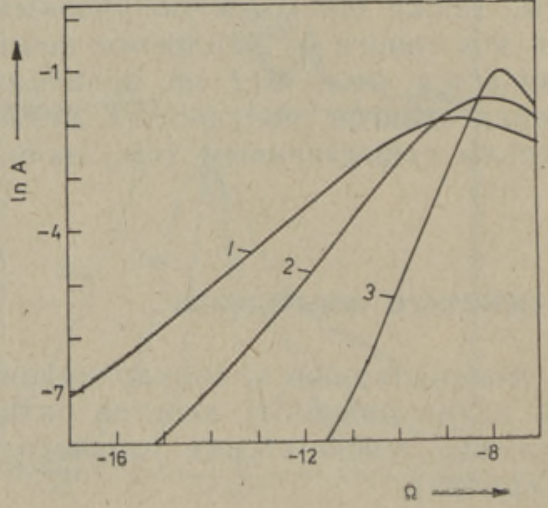

Рис. 8. Длинноволновые участки спектра поглощения в случае $B=$ $=12, S=7$ в полулогарифмнческом масштабе прн $T=1,5 \quad(1), T=1,0$ (2) и $T=0,5$ (3).

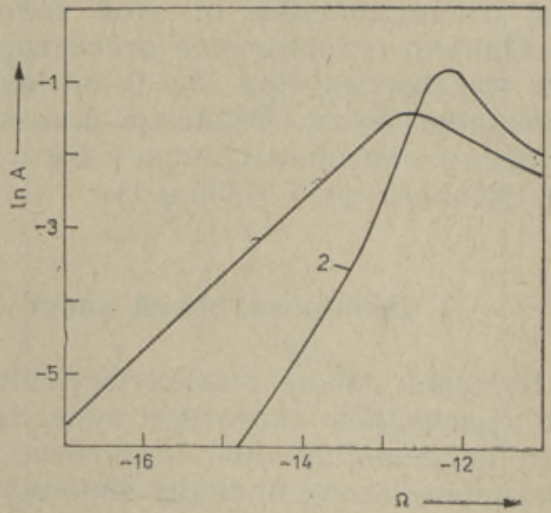

Рис. 9. То же, что и на рис. 8 , при $B=20, S=7, T=1,5$ (I) и $T=1$ (2). 
ных кристаллов с сильным экситон-фононным взаимодействием был ранее предложен в $\left[{ }^{14}\right]$. Длинноволновые хвосты поглощения при более высоких температурах для параметров рис. 6 показаны в полулогарифмическом масштабе на рис. 8. Как видно из этого рисунка, частотное поведение спектров удовлетворяет правилу Урбаха, причем все три кривые имеют общую частоту конвергенции $\Omega_{0} \approx-7,9$ близкую к частоте максимума спектра при $T=0$. Положив в $(11) \omega=1$, находим $\sigma=1,13$ при $T=1$ и 1,5 и $\sigma=1,3$ при $T=0,5$. Отметим, что для параметров рис. 8 , в отличие от обсуждавшегося ранее случая слабой связи, и при нижайшей из рассмотренных температур $T=0,5$ не наблюдается фононная структура типа изображенной на рис. 5.

На рис. 9 показаны в полулогарифмическом масштабе длинноволновые хвосты оптического поглощения в случае $B=20, S=7$. Как видим, и для этих параметров частотное поведение спектра удовлетворяет правилу Урбаха, причем $\sigma=1,6$ для $T=1,5$ и $\sigma=1,9$ для $T=1$. Кривые при $T=1$ на этом рисунке и $T=0,5$ на предыдущем выглядят несколько вогнутыми из-за лоренцевского уширения $\delta$-функций, формирующих спектр (конечной величины $\eta$ в (2)), использовавшегося при получении этих кривых. С уменьшением $\eta$ ход кривых в полулогарифмическом масштабе становится более близким к линейному.

Заметим, что полученные из рис. 8 и 9 значения б приблизительно удовлетворяют соотношению $\sigma=s B / 2 S$, где $s$ - постоянная порядка единицы, предложенному в $\left[{ }^{6,9,14}\right]$. В случае $B=12, S=7 s=1,3-1,5$, при $B=20, S=7 \quad s=1,1-1,3$. Длинноволновой участок спектра при $T=1$ на рис. 5 , несмотря на отмечавшуюся выше фононную структуру, также имеет частотное поведение близкое к описанному формулой (11), причем $\sigma=2,5, s=1,2$. Эти значения близки к величине $s=1,5$, полученной в $\left[{ }^{9}\right]$ в адиабатическом приближении. Сходство результатов позволяет использовать для интерпретации длинноволнового участка спектра при указанных наборах материальных параметров и достаточно высоких температурах представления, следующие из этого приближения: хвост оптического поглощения обусловлен переходами в состояния экситона, локализованные в случайных флуктуациях потенциала, вызванных колебаниями решетки. При этом близкий к экспоненциальному на протяжении нескольких порядков вид спектра (11) в случае трехмерного кристалла обусловлен совместным действием частотных зависимостей плотности этих состояний и силы осциллятора перехода в них $\left[{ }^{9,30}\right]$. Указанные флуктуационные состояния не следует отождествлять с состояниями АЛӘ. Первые существуют при достаточно высоких температурах и в тех случаях, когда состояний АЛЭ нет (см., напр., рис. 9). Кроме того, как уже отмечалось, если последние н существуют, в окрестности максимума СЭ эти состояния могут проявляться в спектре лишь при достаточно низких температурах (рис. 6), значительно более низких, чем на рис. 8. Состояния АЛЭ в этом случае дают вклад в высокотемпературный спектр поглощения в иной спектральной области, $\Omega_{0}-\Omega \geqslant \mathrm{B}$, где радиус возбуждения мал и применима модель с малым числом конфигурационных колебаний (см. также $\left.\left[{ }^{16}\right]\right)$. Возвращаясь к низкотемпературным спектрам при $S \gg 1$, отметим, что и в этом случае можно ожидать появления фононной структуры типа изображенной на рис. 5 на длинноволновом спаде спектра при температурах близких к нулю. Из-за малости поглощения здесь этот вопрос в данной работе не исследовался.

Таким образом, использовавшаяся модель экситон-фононного взаимодействия дает вполне удовлетворительное описание ряда экспериментальных результатов как в центральной области экситонного спектра поглощения некоторых диэлектрических, полупроводниковых и молекулярных кристаллов, так и на его длинноволновом спаде. Примененная 
вычислительная процедура, модифицированный алгоритм Ланцоша, позволяет в едином подходе вычислять корреляционные функции и связанные с ними спектры поглощения в широкой области материальных параметров и температур. Получаемые при этом результаты более точно, чем в других подходах, отражают экспериментальную ситуацию, что позволяет дать интерпретацию наблюдающимся особенностям и предсказать некоторые новые.

Автор признателен В. Хижнякову за полезное обсуждение работы.

\section{Л И Т Е Р А Т Р А}

1. Toyozawa, Y. Progr. Theor. Phys., 20, № 1, 53-81 (1958).

2. Toyozawa, Y. Progr. Theor. Phys., 27, № 1, 89-104 (1962)

3. Москаленко С. А., Шмиглюк М. Н., Чиник Б. Н. ФТТ, 10, вып. 2, 351-358 (1968).

4. Ницович Б. М., Фаленчук В. Д. УФЖ, 21, № 6, 904-910 (1976).

5. Клокишнер С. И., Перлин Ю. Е., Цукерблат Б. С. ФТТ, 20, вып. 11, 3201-3210 (1978).

6. Sumi, H. J. Phys. Soc. Japan, 32, № 3, 616-628 (1972).

7. Sumi, H. J. Phys. Soc. Japan, 36, № 3, 770-779 (1974); 38, № 3, 825-835 (1975).

8. Miyazaki, H., Hanamura, E. J. Phys. Soc. Japan, 50, № 4, 1310-1320 (198i).

9. Schreiber, M., Toyozawa, Y. J. Phys. Soc. Japan, 51, № 5, 1528-1536, 1537-1543, $1544-1550$ (1982).

10. Sherman, A. V. Phys. status solidi (b), 135, № 2, 697-705 (1986).

11. Парлет Б. Симметричная проблема собственных значений. М., «Мир», 1983.

12. Sherman, A. V. Phys, status solidi (b), 131, № 1, 225-233 (1985).

13. Kurik, M. V. Phys. status solidi (a) 8, № 1, 9-45 (1971).

14. Sumi, H., Toyozawa, Y. J. Phys. Soc. Japan, 31, № 2, 342-358 (1971).

15. Segal, B. Phys. Rev., 150, № 2, 734-742 (1966).

16. Носелевич А. С. ЖЭТФ, 83, № 2(8), 743-755 (1982).

17. Sherman, A. V. J. Phys. A, 20, № 3, 569-576 (1987)

18. Mori, H. Progr. Theor. Phys., 34, № 3, 399-416 (1965).

19. Nagano, K., Karasudani, T., Okamoto, H., Mori, H. Progr. Theor. Phys., 63, № 6, 1904-1916 (1980).

20. Howard Lee, M. Phys. Rev. B, 26, № 5, 2547-2551 (1982).

21. Ребане K. K. Элементарная теория колебательной структуры спектров примесных центров кристаллов. М., «Наука», 1968.

22. Ткач Н. В., Лопушанская Н. В., Валь А. Д. Изв. вузов. Физика, 29, № 10, 93-98 (1986).

23. Рашба Э. И. Оптика и спектр., 2, № 1, 75-87, 88-98 (1957).

24. Завт Г. С., Рейфман С. П., Шуличенко Б. В. ФТТ, 22, вып. 3, $841-848$ (1980).

25. Hizhnyakov, V., Kink, R., Selg, M., Sherman, A. In: Ultrafast Phenomena in Spectroscopy (2nd Intern. Conf.). Reinhardsbrunn (GDR), 1980, 468-472.

26. Baldini, G., Bosacchi, A., Bosacchi, B. Phys. Rev. Lett., 23, № 15, 846-848 (1969).

27. Baldini, G., Bosacchi, B. Phys. Rev. Lett., 22, № 5, 190-192 (1969).

28. Луцик Ч. Б. В кн.: Экситоны (ред. Э. И. Рашба, М. Д. Стердж). М., «Наука», $1985,362-384$.

29. Tomiki, T., Miyata, T., Tsukamoto, H. J. Phys. Soc. Japan, 35, № 2, 495-507 (1973).

30. Ihm, J., Phillips, J. C. Phys. Rev. B, 27, № 12, 7803-7806 (1983).
Институт физики
Академии наук Эстонской ССР
Поступила в редакцию 1/VI 1987

\section{A. SERMAN \\ EKSITONNEELDUMISSPEKTRITE SŌLTUVUS TEMPERATUURIST. ARVUTUSED REKURSIIVMEETODIL}

Korrelatsioonfunktsioonide arvutamise rekursiivmeetodi alusel on uuritud dispersioonivaba optilise foononharuga kontaktselt interakteeruvate Frenkeli eksitonide tsooni sisaldava kolmemõōtmelise dielektrilise kristalli mudeli neeldumisspektreid laias aine parameetrite ja temperatuuride piirkonnas. Käsitletud on eksitoni ja foononi nõrka seost, vibroonse eksitoni ja peaaegu vaba ning autolokaliseeritud eksitoni kooseksisteerimise juhtumeid, samuti vahepealseid situatsioone, millele vastavad mitmekesise kujuga spektrid. 


\section{TEMPERATURE DEPENDENCE OF THE EXCITON ABSORPTION SPECTRA. CALCULATIONS BASED ON THE RECURSION METHOD}

Based on the recursion method of the calculation of correlation functions, absorption spectra of a model of a three-dimensional crystal containing a band of Frenkel excitons interacting with a branch of dispersionless optical phonons, are investigated in a wide range of material parameters and temperature. The cases of a weak exciton-phonon coupling, vibronic exciton, coexistence of nearly free and self-trapped excitons as well as intermediate situations which a wide variety of spectral shapes corresponds to, are considered. It is shown that in a definite region of parameters the character of exicitonphonon coupling is changed with the growth of temperature such that a spectrum revealing the features of a weak coupling is transformed to a curve similar to the vibronic exciton spectrum. In addition, in a definite temperature range, a change in the character of observed peculiarities occurs - the main exciton maximum is transformed into one of the phonon repetitions and, conversely, one of such repetitions is converted into the main peak. In the case of coexistence of nearly free and self-trapped states the temperature behaviour of the peculiarities connected with these two types of excitations are shown to be different, a fine structure caused by self-trapped states being manifested in the vicinity of the main peak only in the low-temperature spectra. At higher temperatures a spectral shape can essentially differ from the asymmetric Lorentzian due to a sideband on a short-wave side of a maximum. A long-wave absorption tail at high temperatures and considered sets of parameters satisfies the Urbach rule. With lowering of temperature in the case of a weak exciton-phonon coupling, a phonon structure with the step approximately equal to the phonon frequency stands out in this tail, 